\title{
Wall conditioning at the Wendelstein 7-X stellarator operating with graphite divertor
}

\author{
A. Goriaev ${ }^{1,2}$, T. Wauters ${ }^{1}$, R. Brakel ${ }^{3}$, S. Brezinsek ${ }^{4}$, A. Dinklage ${ }^{3}$, J. Fellinger ${ }^{3}$, H. \\ Grote $^{3}$, D. Moseev ${ }^{3}$, S. Sereda ${ }^{4}$, O.Volzke ${ }^{3}$ and W7-X team \\ ${ }^{1}$ Laboratory for Plasma Physics, LPP-ERM/KMS, Brussels, Belgium \\ ${ }^{2}$ Department of Applied Physics, Ghent University, Belgium \\ ${ }^{3}$ Max-Planck-Institute for Plasma Physics, Greifswald, Germany \\ ${ }^{4}$ Institute for Energy and Climate Research - Plasma Physics, Forschungszentrum Jülich GmbH, Jülich, \\ Germany
}

E-mail: a.goriaev@fz-juelich.de

Received $\mathrm{xxxxxx}$

Accepted for publication $\mathrm{xxxxx}$

Published xxxxxx

\begin{abstract}
Wall conditioning plays an important role in achieving record plasma performance on superconducting stellarator Wendelstein 7-X (W7-X) by controlling plasma density and keeping low level of impurities. The development of the optimal wall conditioning strategy is of high importance for successful operation of W7-X with graphite divertor. The current strategy consists of initial wall conditioning applied prior to physics experimental program and wall conditioning techniques executed during plasma operation phase. The combination of baking and glow discharge cleaning (GDC) provides fast start of reliable plasma operation. Regular boronisation opens a new operational window of high plasma densities above $1 * 10^{20}$ $\mathrm{m}^{-3}$. Strong wall fueling remains however one of the main issues preventing plasma density control. A reliable solution is provided by application of He ECRH pulse trains which are optimised to maximise fuel removal.
\end{abstract}

Keywords: W7-X, wall conditioning, He pulse train, boronisation, GDC, baking, ECWC

\section{Introduction}

The optimized superconducting stellarator W7-X (with its major radius of $5.5 \mathrm{~m}$ and minor radius of $0.5 \mathrm{~m}, 2,5 \mathrm{~T}$ of magnetic field on axis and $30 \mathrm{~m}^{3}$ of plasma volume), built to demonstrate its capability of steady-state plasma operation, started its first operation in divertor configuration in 2017 [1]. In this configuration $\mathrm{W} 7-\mathrm{X}$ was equipped with inertially cooled stainless steel wall panels, graphite heat shields and 10islands graphite divertor units. This stage of W7-X operation was determined by maximum heating energy of $200 \mathrm{MJ}$ and pulse length of up to $100 \mathrm{~s}$ [2]. Aiming to achieve high performance plasmas this operational stage was split in two experimental campaignes (OP1.2a and $\mathrm{OP} 1.2 \mathrm{~b})$. It required the control of fuel recycling and minimization of impurity release from the first wall during plasma experiments. Wall conditioning has already been proven to be effective to control the surface state of Plasma Facing Components (PFC) of W7$\mathrm{X}$ [3]. Different wall conditioning techniques are applied to achieve the complex aim of high plasma performance. The wall conditioning strategy comprises two stages. The initial conditioning, prior to the first plasma, includes baking of the vacuum vessels and glow discharge cleaning (GDC) to provide minimum conditions to start physics experiments by decreasing the amount of water, hydrocarbons and methane in the residual gas content. Boronisation and He ECRH pulse 
trains were used during the plasma operaration period to reduce the amount of oxygen and control the hydrogen recycling, respectively.

This paper gives a brief description of each wall conditioning stage with optimisation of the included conditioning techniques. It also summarises the results of application and optimisation in the current wall conditioning strategy which is relevant for W7-X operation with graphite divertor.

\section{Wall conditioning techniques}

\subsection{Baking}

The bake-out of the inner walls was done in each operational phase after the pump-down of the plasma vessel. The whole procedure of baking was conducted during 11 days, including a flat top phase of 7 days where the average wall temperature was maintained at $150{ }^{\circ} \mathrm{C}$. A detailed technical discription of baking procedure for different elements of the $\mathrm{W} 7-\mathrm{X}$ vacuum vessel is given in [4].

The residual gas pressure trends during the first conditionining step show the same behaviour for two experimental campaigns in divertor configuration. As it was previously discussed [5] outgassing during the flat top phase follows a $\sim t^{-0.7}$ time dependence. Thus, while the duration is experienced satisfactory, this dependency implies that a short extension of the bake out time would bring only minor further improvements. Baking allowed to decrease the base level of pressure from $(8.1 \pm 0.6)^{*} 10^{-8}$ mbar to $(3.9 \pm 0.4) * 10^{-8} \mathrm{mbar}$ prior to OP1.2a and from $(5.4 \pm 0.8)^{*} 10^{-8}$ mbar to $(2.1 \pm$ $0.4)^{*} 10^{-8}$ mbar prior to OP $1.2 \mathrm{~b}$.
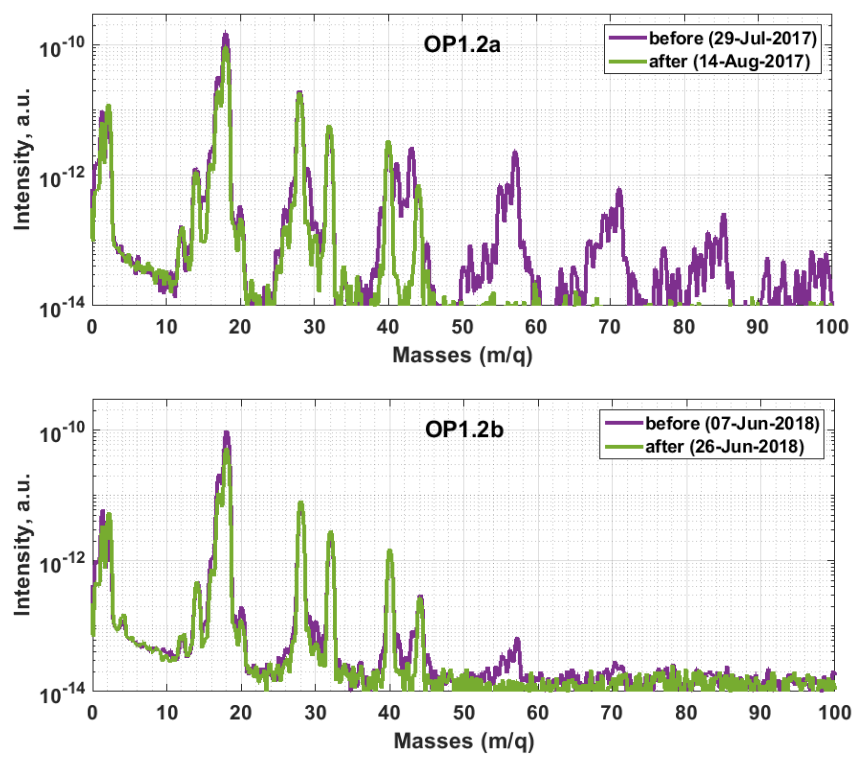

Fig. 1 Residual gas content before (magenta line) and after (green line) baking: top - OP1.2a (01-Aug-2017 - 12-Aug-2017), bottom - OP1.2b (11Jul-2018 - 23-Jul-2018).
The results of baking for both experimental campaigns are shown in Fig.1 by comparison of residual gas content. The data was recorded by Quadrupole Mass Spectrometer (QMS) Pfeiffer PrismaPlus QMG 220. Two mass spectrometers were located in the pumping ducts approximately 3 meters away from the vacuum vessel. Both devices were differentially pumped and measurements were done at the room temperature. The data was recorded with a systematic error of $2-4 \%$. The QMS spectra were not absolutely calibrated to the different gas partial pressures.

According to the comparison of the mass-spectra recorded before and after baking large removal of impurities was observed in OP1.2a. The outgassing of all heavy species with mass-to-charge ratio above 50, mostly high hydrocarbons, was suppressed. Water, as being the dominating impurity, was reduced in the residual gas by factor of $1.6 \pm 0.2$. The amounts methane $\left(\mathrm{CH}_{4}\right)$ and ethane $\left(\mathrm{C}_{2} \mathrm{H}_{6}\right)$ were decreased by factor of $1.6 \pm 0.1$ and $2.1 \pm 0.2$, respectively. However, partial pressures of these species are approximetaly 2 orders of magnitude lower than water partial pressure. In case of air related species such as oxygen $\left(\mathrm{O}_{2}\right)$, carbon dioxide $\left(\mathrm{CO}_{2}\right)$ and argon (Ar) there were almost no changes. The identification of a change of $\mathrm{CO}$ as an oxygen containing impurity with the corresponding mass-to-charge ratio of 28 is not straightforward. Nitrogen $\left(\mathrm{N}_{2}\right)$, ethane $\left(\mathrm{C}_{2} \mathrm{H}_{6}\right)$ and some fractions of $\mathrm{CO}_{2}$ can also contribute to this QMS signal [6]. The mass-to-charge ratio of 28 signal has no significant difference. That correlates with behaviour of signals related to mass-to-charge ratio of 14 according to the cracking patterns [6]. Thus, there is only the strong contribution of $\mathrm{N}_{2}$ to massto-charge ratio of 28. Amount of $\mathrm{CO}$ and its changes are negligible.

In comparison with the first divertor campaign baking prior to OP1.2b did not lead to such a dramatic change of the impurity content. Partial pressures of $\mathrm{N}_{2}, \mathrm{O}_{2}, \mathrm{CO}_{2}$ and $\mathrm{Ar}$ remained unchanged. The water level was decreased by factor of $1.9 \pm 0.1$. Another significant change in the residual gas content was a decrease ( 1.5 times) of $\mathrm{CH}_{4}$ level wich concentration was $1.3 \pm 0.3 \%$ of water concentration prior to OP1.2b. Thus, OP1.2b baking removed mostly water. It is also due to the fact that the wall conditions prior OP1.2b were significantly better already after the pump down. The larger impurity content prior to OP1.2a is due to the longer venting and in-vessel assembly time ( 14 months before OP1.2a vs. $\sim 4$ months before OP1.2b).

\subsection{Glow discharge cleaning}

Glow discharge cleaning (GDC) was applied in hydrogen and helium before and sometimes in between plasma experiment operation days. GDC in hydrogen was used to reduce the remaining part of the dominating impurities $\left(\mathrm{H}_{2} \mathrm{O}\right.$, $\mathrm{CH}_{4}, \mathrm{CO}_{2}$ ) which could not be further removed by baking (Fig.1). It also helped to further improve the wall conditions 
throughout the experimental campaign and to delete some residual gas species formed during plasma operation. GDC in helium was done in order to desaturate the walls from hydrogen after either $\mathrm{H}_{2}-\mathrm{GDC}$ or $\mathrm{H}_{2}$ - plasma experiments.

The glow discharge system is equipped with 10 graphite anodes operated at maximum current of $1.5 \mathrm{~A}$ (within $3 \mathrm{~kW}$ ) for each unit [7]. The optimization of GDC parameters was done in OP1.2a. Aiming to increase the cleaning efficiency, shorten the execution time and minimize the erosion of PFC during the ignition phase of GDC the following optimal parameters have been defined [8]. For glow discharge in hydrogen working pressure was $(4.5 \pm 0.1)^{*} 10^{-3}$ mbar at maximum allowed anode current of $1.5 \mathrm{~A}$ for all 10 anodes separately, the average voltage was $\sim 305$ V. Pressure of (3.8 $\pm 0.1)^{*} 10^{-3}$ mbar and anode current of 1 A corresponding to average voltage of $210 \mathrm{~V}$ were found optimal for He-GDC. The summary of GDC operation thoughout the divertor phase is given in Table 1.

\begin{tabular}{c|cc}
\multirow{2}{*}{$\begin{array}{c}\text { Operational } \\
\text { Phase }\end{array}$} & OP1.2a & OP1.2b \\
\cline { 2 - 3 } & \multicolumn{2}{|c}{ Prior to first plasma } \\
\hline $\mathbf{H}_{2}-$ GDC & $2: 58 \mathrm{~h}$ & $9: 35 \mathrm{~h}$ \\
He- GDC & $0: 36 \mathrm{~h}$ & $1: 50 \mathrm{~h}$ \\
\hline & \multicolumn{2}{|c}{ During plasma operation } \\
\hline $\mathbf{H}_{2}-$ GDC & $13: 46 \mathrm{~h}$ & $1: 03 \mathrm{~h}^{*}$ \\
He- GDC & $14: 48 \mathrm{~h}$ & $2: 24 \mathrm{~h}^{*}$
\end{tabular}

Table 1. Glow discharge cleaning throughout inertially cooled divertor operational phase. The short time of GDC during plasma operation in $\mathrm{OP} 1.2 \mathrm{~b}$ is determined by early boronisation. *before boronisation, excluding GDC in $\mathrm{He} /$ diborane mixture

In OP1.2a the initial GDC session was relatively short. In contrast, GDC was actively used, in hydrogen - weekly and in helium - daily before the physics program, throughout the experimental campaign. $\mathrm{H}_{2}-$ GDC has shown its cumulative effect of impurity removal in OP1.2a [9]. The study shows that most of impurity trends follow $\sim t^{-0.7}$ time dependence except methane and carbon dioxide. This exception can be due to dominating chemical sputtering processes. Thus, in order to achieve an impurity level reduction of more than one order of magnitude and achieve reliable plasma operation conditions at least $9-10$ hours of $\mathrm{H}_{2}$-GDC have to be performed at the initial wall conditioning phase. That was done before the plasma operation of OP1.2b. Almost 9.2 hours of $\mathrm{H}_{2}$-GDC was applied within the initial conditioning phase splitted in two sessions with a break of 1 week. The behaviour of impurity reduction in that case has also proved the cumulative effect of $\mathrm{H}_{2}$-GDC [5]. 1 hour and 50 minutes of He-GDC were applied to desaturate the PFC surfaces from hydrogen achieving the required hydrogen outgassing level allowing a reliable start of the following plasma experiments.

In comparison to the first divertor campaign, GDC was routinely applied only in the beginning of OP1.2b. This procedure was suspended after the first boronisation (i) due to improved wall conditions and (ii) in order to avoid possible erosion of the boron layer during the GDC. Due to the improved conditions after boronisation, density control could hereafter be done only by ECRH wall conditioning in helium.

\subsection{Boronisation}

The application of boronisation on W7-X became possible in OP1.2b. The aim of boronisation was to minimize the amount of oxygen in plasma and, therefore, to reduce its radiative and sputtering activity. Three boronisations were done with an interval time of one month. Each boronisation was based on glow discharge application in the mixture of helium and diborane (90:10) with an active coating phase of $3.5-5.5$ hours. The procedure was followed by short He-GDC for residual hydrogen and diborane removal. The glow discharge on W7-X is inhomogeneously distributed. The current density at the wall varies with the distance from glow anodes [10]. Due to this, the boron coating is assumed to be not uniformly distributed on the PFC. The averaged thickness of boron layer is estimated preliminary around $10 \mathrm{~nm}$. This number assumes that all injected boron ends up on the wall with surface area of $110 \mathrm{~m}^{2}$, and forms a pure amorphous boron layer with density $2.4 \mathrm{~g}^{*} \mathrm{~cm}^{-3}$. However, the experience of boronisations at similar conditions on TEXTOR [11] and W7-AS [12] shows that the average thickness of boron layer can reach $100 \mathrm{~nm}$. Nevertheless, more accurate numbers will be available after PFC post-mortem analysis and modeling of boronisation process on $\mathrm{W} 7-\mathrm{X}[13,14]$.

The first boronisation was applied three weeks after the beginning of the plasma operation. Its impact on wall conditions and plasma performance was observed by comparison of the series of density ramp hydrogen reference discharges (4 MW of ECRH power, $6 \mathrm{~s}$, standard magnetic configuration [15]) executed before and after the born coating procedure.

The most valuable change brought by boronisation was establishing stable hydrogen plasma operation in the wide density range from $\sim 1 * 10^{19} \mathrm{~m}^{-3}$ to more than $1 * 10^{20} \mathrm{~m}^{-3}[16]$. The significant improvement of plasma performance has been achieved due to the reduction of main radiators among plasma impurities such oxygen and carbon. Indeed, the dramatic drop of oxygen and carbon level in plasma discharges after the first boronisation was observed by various diagnostics [17]. For example, fluxes of oxygen and carbon atoms towards the divertor were reduced by factors of 10 and 8 , respectively [1].

The analysis of mass-spectra of the outgassed content during density ramp reference discharges also shows that the production of water and carbon oxides is lower after the first boronisation. This difference in outgassing trends is given on Fig.2. The relative amount of presented impurities was derived from the QMS spectra according to the cracking patterns given in [6]. Particularly, the mass-to-charge 28 signal for $\mathrm{CO}$ was corrected for possible contributions of $\mathrm{N}_{2}$ by using the massto-charge 14 time trace. The comparison of outgassing curves 
for subsequent reference dischages shows the decrease of $\mathrm{CO}$, $\mathrm{CO}_{2}$ and $\mathrm{H}_{2} \mathrm{O}$ by factors of $9.3 \pm 0.9,2.7 \pm 0.7$ and $1.7 \pm 0.2$, respectively. These numbers were calculated by taking an average between (i) outgassing peaks ratios of the reference discharges and (ii) integrals of the outgassing curves after discharge terminations. The rise of the water release $100 \mathrm{~s}$ after the pre-boronisation reference discharge (blue curve) is caused by the strong heating of the divertor plates above $\sim 650$ ${ }^{0} \mathrm{C}$ according to divertor termocouples measurements. That leads to outgassing of water contained in the deep layers of fine grain graphite [17]. It is not assumed to have an influence on the data comparison. The formation of $\mathrm{CO}$ and $\mathrm{CO}_{2}$ during plasma operation is mostly due to the process of chemical sputtering of carbon PFC by oxygen [18]. Thus, boronisation, which reduced the oxygen content in the plasma, also leads to mitigation of graphite PFC erosion by oxygen, and subsequent selfsputtering by carbon. A different picture is observed for methane production. According to given mass-spectra analysis boronisation has almost no influnce on this process (Fig.2).
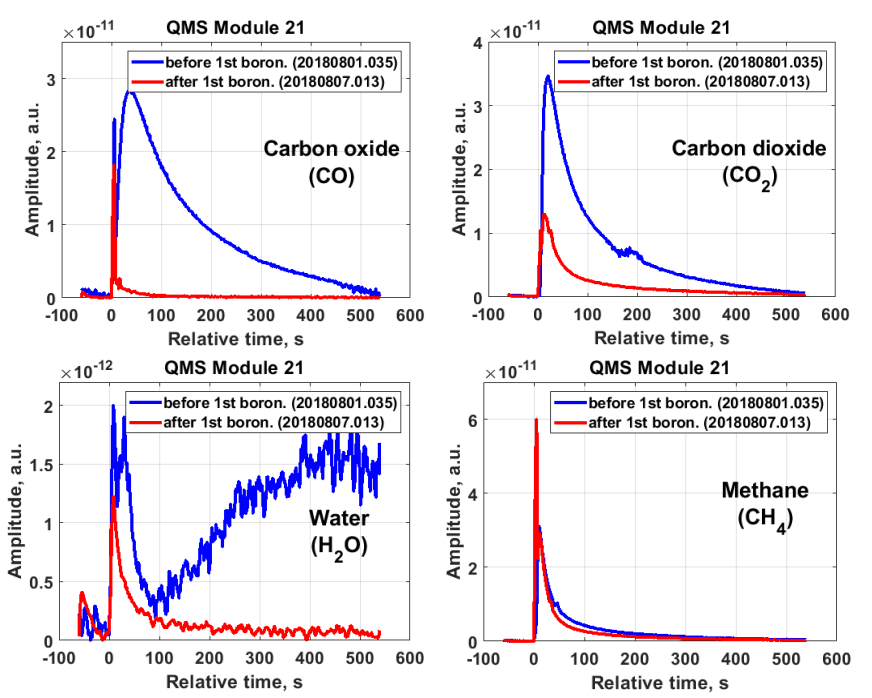

Fig. 2 Partial pressures of carbon and oxygen based impurities before and after $1^{\text {st }}$ boronisation calculated from QMS recorded data during density ramp reference discharges. Blue curves indicate outgassing during the reference discharge (20180801.035) before the first boronisation, red curves belong to partial pressures during the reference discharge (20180807.013) after the first boronisation.

One of the outstanding questions is the life-time of the deposited boron layer. Even though that the boron was almost removed from the strike point area after $\sim 200 \mathrm{~s}$ of subsequent plasma [2], giving rise to a local increase of carbon and oxygen radiation in the different areas of divertor units, the oxygen level measured at the carbon heat shield by a filterscope has never reached pre-boronisation values. Moreover, the high plasma performance did not degrade significantly between boronisations. VUV spectroscopic observation (HEXOS diagnostic [19]) has also shown that carbon and oxygen concentrations did not have a significant variation in plasma core between boronisations.

These facts point out that the extension of periods between boronisation can be done without compromising the high plasma performance at similar device configurations.

\subsection{He Electron Cyclotron Wall Conditioning}

In spite of the evident plasma performance improvement brought by boronisation, the problem of plasma density control was not fully solved. Strong wall fueling remained an issue when changing operation from high density (above $\left.1 * 10^{20} \mathrm{~m}^{-3}\right)$ to low density discharges $\left(3-5 * 10^{19} \mathrm{~m}^{-3}\right)$ in hydrogen in a daily physics program.

The activated magnetic field prevents the application of glow discharge to sustain the density control throughout of the experimental day. Thus, only Electron Cyclotron Wall Conditioning (ECWC), standard $140 \mathrm{GHz}$ X2 ECRH helium plasma discharges, could be used as a conditioning tool for desaturating the wall from hydrogen between main plasma discharges. In OP1.2a it was shown that application of $\mathrm{He}$ pulse trains, sequences of short He discharges with certain pulse length, pulse interval and moderate input power, is more efficient and reliable than the use of so-called He recovery discharges, long high energy discharge in helium (up to $10 \mathrm{~s}$ and $30 \mathrm{MJ}$ ) [8]. He pulse trains were continuously optimised in terms of pulse length, input power, gas prefill and interval between pulses throughout the first campaign of divertor operational phase of W7-X by comparison of its hydrogen removal efficiency. Boronisation minimized the risk of radiative collapses during the application of He cleaning pulses. This allowed to extend the parameter range in pulse optimisations compared to previous studies.
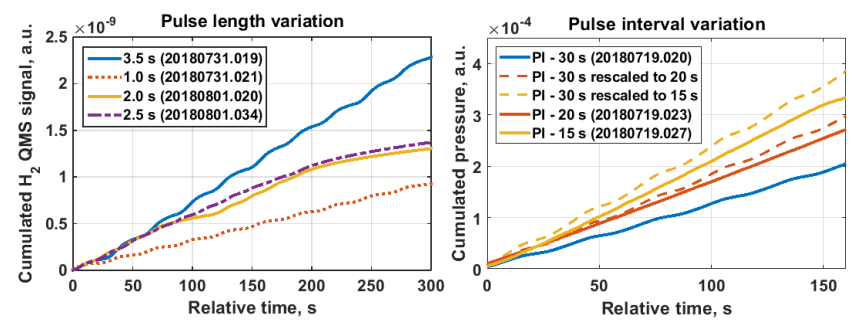

Fig. 3 He pulse train optimization by variation of pulse length (left) and pulse interval (right). The cleaning efficiency is shown by cumulated hydrogen removal calculated from the partial pressure measurements of Quadrupole Mass-Spectrometer (QMS). Relative time equal 0 indicates the beginning of the first pulses. Dashed lines show predicted hydrogen removal for $30 \mathrm{~s}$ pulse interval rescaled to pulse intervals of $20 \mathrm{~s}$ and $15 \mathrm{~s}$.

Fig.3 shows the comparison of the hydrogen removal efficiency in pulse trains with different pulse length and pulse interval. As a reference for the initial conditions for each of pulse sequences the first pulses of each squence were identical. The hydrogen QMS strace for each sequences was then rescaled with a constant factor such that the hydrogen removal after these first pulses is idendentical. Pulse lengths 
from 1 to $3.5 \mathrm{~s}$ are compared at constant parameters of input power $(2.1 \mathrm{MW})$ and pulse interval $(20 \mathrm{~s})$. It is clearly seen that the removal of hydrogen increases with the longer pulse length. However, the dependence is not linear and further analysis including fuel redeposition rates is needed to make the full description.

Next, a further reduction of the pulse interval compared to [8] was tested in OP1.2b. To better illustrate the difference in the cumulated removal traces, the curve of the pulse train (20180719.020) with a $30 \mathrm{~s}$ pulse interval was rescaled to the expected cumulative outgassing traces at pulse intervals of 20 $\mathrm{s}$ and $15 \mathrm{~s}$, by multiplying by ratio $(P I+P L) /\left(P I_{\text {new }}+P L\right)$, where $P L$ is a pulse length, $P I$ is a pulse interval of $30 \mathrm{~s}$ and $P I_{\text {new }}$ is a new pulse interval $(20 \mathrm{~s}$ or $15 \mathrm{~s})$. Experimentally, the reduction of pulse interval down to $15 \mathrm{~s}$ leads to a slight increase of time averaged removal rates (Fig.3 left). However, it is less than predicted hydrogen removal due to hydrogen redeposition. The characteristic pumping time for hydrogen $t_{0}$ $=\mathrm{V} / \mathrm{S}$ on $\mathrm{W} 7-\mathrm{X}$ is $3.3 \mathrm{~s}$, with $\mathrm{V}$ the vessel volume and $\mathrm{S}$ the hydrogen pumping speed. The typical observed pressure decay time constant after a discharge is however higher, about $\sim 7.0 \mathrm{~s}$, due to continues outgassing from the PFC's. While a pulse interval of $15 \mathrm{~s}$ allows still to evacuate most of the wall released hydrogen before the next pulse. Reducing the pulse interval even further will bring only minor improvement to the time averaged removal rate as redeposition of wall released hydrogen will become more significant. Moreover, heavier impurities will have even stronger accumulation and redeposition rates due to lower pumping speed. Thus, it was decided to keep $30 \mathrm{~s}$ of pulse interval as it was used in the beginning of OP1.2b. Moreover, this slightly larger pulse interval increases the reliability of the ECRH system operation.

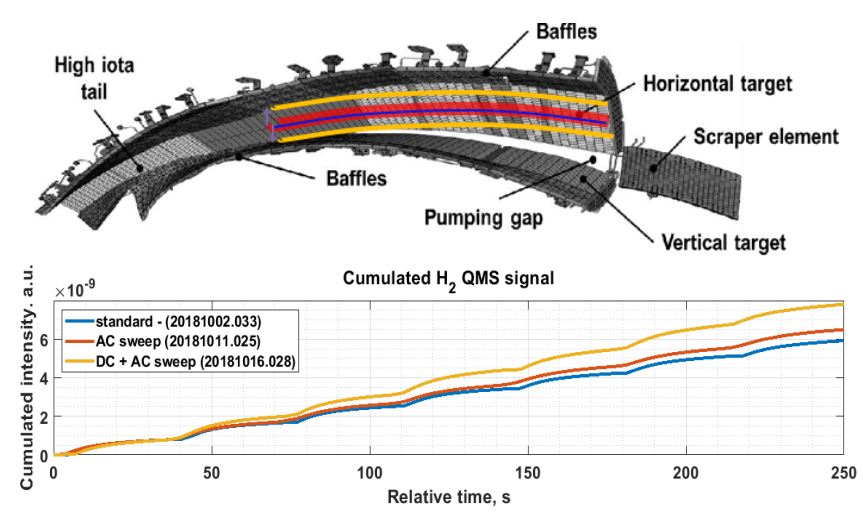

Fig.4 (top) The sketch of the divertor unit and different total plasma wetted area shown by different colors corresponded to the standard pulse train (blue line), the pulse train with AC sweep (red line) and the pulse train with DC + AC sweep (yellow line). (bottom) Cumulated removal of hydrogen by three He pulse trains with main identical parameters: standard (blue line), AC sweep (red line), DC + AC sweep (yellow line).

An additional improved He pulse train scheme which became available in OP1.2b was sweeping of the strike lines during the execution of pulse sequences. The shift of strike lines across the target surface of the divertor could be done by using the control coils. The application of AC (alternating current) with an amplitude of up to $625 \mathrm{~A}$ and frequency of 20 $\mathrm{Hz}$ (AC sweep, during a pulse) or up to +/- $2500 \mathrm{~A}$ of DC (direct current), so-called DC sweep (from pulse to pulse), on the control coils allows to manipulate the poloidal position and behaviour of the strike lines [20]. Moreover, the strike line sweeps described above can be also combined (DC $+\mathrm{AC}$ sweep) when AC and DC are applied simultaneously.

Fig. 4 shows the result of one of the strike line sweeping experiments. The investigation of strike lines sweep influence on hydrogen removal was performed by analysis of three pulse trains with identical main parameters (pulse length of $3 \mathrm{~s}$, pulse interval $35 \mathrm{~s}$, input power of 2.1 MW). The first pulse train was without sweeping, so-called standard pulse train. Two others were performed with AC sweep (625 A and 20 $\mathrm{Hz}$ ) and $\mathrm{DC}+\mathrm{AC}$ sweep (from pulse to pulse DC amplitude was changed from 0 to $+/-1250 \mathrm{~A}$ and $+/-2500 \mathrm{~A}$ and $\mathrm{AC}$ had $625 \mathrm{~A}$ and $20 \mathrm{~Hz}$ ). The application of AC sweep leads to the oscillation of the strike lines around its standard positions. Positive or negative DC values shift a strike line to a new position [21]. Subsequently, total plasma wetted area increases when the additional strike line sweep option is added (Fig.4 top). According to results of experiment shown in Fig. 4 (bottom) one can see that the pulse train with AC sweep removes a bit more hydrogen in comparison with standard pulse trains. At the same time, the use of $\mathrm{DC}+\mathrm{AC}$ sweep gives significant improvement to pulse train cleaning efficiency. Up to $30 \%$ more of removed hydrogen can be gained applying this type of strike line sweeping. Thus, the fuel removal depends on the total plasma wetted area on the divertor units. It should be also mentioned that during the standard plasma operation the strike line position is very sensitive to the net toroidal current [22]. The strong toroidal current ( $\sim 5 \mathrm{kA})$ leading to a strike line movement (up to $3 \mathrm{~cm}$ ) along the target surface can be developed in the order of $10 \mathrm{~s}$ [23]. Thus, the main reservoir of retained fuel can be spread on the divertor target. Therefore, it is preferable to use pulse trains with sweeping after application of long high density discharges because, in this case, the cleaning covers more divertor area.

\section{Summary}

The current wall conditioning strategy for W7-X operating with inertially cooled graphite divertor consists of two parts. The first part is initial wall conditioning done prior to plasma operation. Initial set of wall conditioning starts with 11 days of the vacuum vessel baking. The flat top phase of baking is done during 7 days at average temperature of $150{ }^{0} \mathrm{C}$ to suppress high hydrocarbons and remove water. Glow discharge cleaning (GDC) is the following step. Initial GDC should be done in hydrogen with duration of at least 9-10 hours to reduce the amount of carbon oxydes, methane and water. The last element in the initial conditioning scheme is 
application of 2 hours He-GDC for wall desaturation from hydrogen. The second part of the conditioning strategy is applied during plasma operation. The use of monthly boronisation was sufficient to sustain high plasma performance. Although, the frequency of boronisation should be reduced in following experimental campaigns. After boronisation the routine use of GDC can be skipped. To reduce the wall fueling ECRH He pulse trains are used. Equipped with strike line sweeping optimised He pulse trains can help to achieve the density control faster.

The next step to upgrade the current wall conditioning strategy will be achieved by expoloitation of a new conditioning tool based on Ion Cyclotron Resonance Heating System [24]. It is expected that Ion Cyclotron Wall Conditioning (ICWC) may be more effective for density control than currently used ECWC, as already demonstrated on WEGA [25].

\section{Acknowledgements}

This work has been carried out within the framework of the EUROfusion Consortium and has received funding from the Euratom research and training programme 2014-2018 and 2019-2020 under grant agreement No 633053. The views and opinions expressed herein do not necessarily reflect those of the European Commission.

\section{References}

[1] R.C. Wolf et al. 2019 Phys. Plasmas 26082504

[2] T. Klinger et al. 2019 Nucl. Fusion 59112004

[3] T. Wauters et al. 2018 Nucl. Fusion 58066013

[4] H.-S. Bosch et al. 2017 Nucl. Fusion 57116015

[5] T.Wauters et al. 2019 Plasma Phys. Control. Fusion (accepted PPCF-102660.R1)

[6] J. H. Gross. 2011 Mass Spectrometry. Springer Science \& Business Media

[7] A. Spring et al. 2011 Fusion Eng. Des. 861933

[8] A. Goriaev et al. 2019 Nuclear Materials and Energy 18227

[9] T. Wauters et al. 2018 Nuclear Materials and Energy 17235

[10] A. Canton et al. 2019 Nuclear Materials and Energy 19 468

[11] J. Winter et al. 1989 J. Nuclear Materials 162-164 713

[12] U. Schneider et al. 1992 Fusion Technology 376

[13] C. Li et al. 2019 Spectrochimica Acta Part B: Atomic spectroscopy 160105689

[14] J. Oelmann et al. 2019 Nuclear Materials and Energy 18 153

[15] T. Andreeva et al., 2002 Plasma Phys. 445

[16] T. Sunn Pedersen et al. 2019 Nucl. Fusion 59096014

[17] E. Wang et al. 2019 Phys. Scr. (accepted PHYSSCR109170.R2)

[18] V. Phillips. 2004 Transactions of Fusion Science and Technology $\mathbf{4 5} 237$

[19] W. Biel et al., 2004 Rev. Sci. Instrum. 753268

[20] E. Jauregi et al. 2003 Fusion Eng. Des. 66-68 1125
[21] M. Ślęczka et al. $201845^{\text {th }}$ EPS Conf. on Plasma Physics P2.1020 (http://ocs.ciemat.es/EPS2018PAP/pdf/P2.1020.pdf)

[22] R. C. Wolf et al. 2019 Plasma Phys. Control. Fusion 61 014037

[23] Y. Gao et al. 2019 Nucl. Fusion 59066007

[24] B. Schweer et al. 2017 Fusion Eng. Des. 123303

[25] T. Wauters, et al. 2014 AIP Conf. Proc. 1580187 